\title{
Cohesive energy, stability and structural transitions in polyelectrolyte bundles
}

\author{
Joseph Rudnick \\ Department of Physics and Astronomy, UCLA, Box 951547, Los Angeles, CA 90095-1547 \\ David Jasnow \\ Department of Physics and Astronomy, University of Pittsburgh, Pittsburgh, PA 15260
}

(Dated: November 19, 2018)

\begin{abstract}
A lattice of uniformly charged, infinitesimally thin, rods decorated with an ordered array of counterions exhibits anomalous behavior as the spacing between the rods is varied. In particular, the counterion lattice undergoes a sequence of structural shearing, or "tilting," phase transformations as the spacing between the rods decreases. The potential implications of this behavior with respect to the packaging of biologically relevant polyelectrolytic molecules are commented upon.
\end{abstract}

PACS numbers: $61.20 . \mathrm{Qg}, 61.25 . \mathrm{Hq}, 64.70 . \mathrm{Kb}, 77.80 . \mathrm{Bh}$

\section{INTRODUCTION}

Polyelectrolyte chains naturally repel each other since they carry an overall electric charge. That these chains will nevertheless form condensed phases in the presence of oppositely charged counterions has been known for some time. It has also become clear that this condensation results from some form of organization of the counterions, either dynamical [1, 2] or essentially static, in the form of a counterion lattice [3]. In both cases, the attraction between oppositely-charged regions overcomes Coulomb repulsion between the bare chains and leads to a net attraction. Correlations in the counterion system are crucial, since it is well-known that mean field theory, represented by solution of the Poisson-Boltzmann system of equations, cannot yield an attraction between like-charged rods [1, 4, 5, 6].

The physics of polyelectrolytes is relevant to biological systems. For example, double helix DNA will, under certain conditions, organize into a condensed state in which it self-assembles into bundles of densely packed parallel rods [4, 7]. An important venue for this condensation is within the heads (or capsids) of various viruses [8]. This organization occurs in spite of the fact that "naked" DNA carries a strong negative charge - one excess electron per phsophate group on the backbone [9]. DNA has also been observed to form condensed liquid-crystal-like phases in the presence of polyvalent counterions [10, 11]. In addition, counterion-mediated formation of actin bundles has also been observed [12]. Interestingly, in this last case, there is evidence of a kind of counterion lattice, in the form of a charge-density-wave-like modulation along the axis of the condensed actin filaments. In such a lattice, the "counterions" in the lattice actually consist of clusters of individual counterions, and can therefore not be represented as point particles. The question of an ionic lattice specifically in the case of condensed DNA remains an open one, since there is, as yet, no experimental indication of such an organization of counterions. Furthermore, theoretical estimates indicate that a 3D structure requires counterion valences, $Z$, greater than about $6[3]$. A lattice of point-like counterions in condensed biological rodlike molecules thus remains a conjecture, rather than an established fact. Nevertheless, it represents an sufficiently interesting and potentially important construct within biological and polyelectrolyte physics that its properties merit investigation.

In this paper we analyze more carefully the cohesive energy of the background of negatively charged rods along with a neutralizing, crystalline counterion system. We assume the counterions form a modified face-centered cubic (fcc) crystal. Our principal result is that, as the rod density increases, the counterion system maintains stability by undergoing structural transitions to "tilted" lattices. More precisely, we have located two symmetrybreaking structural transitions: the first is of the threestate Potts type and is therefore weakly first order. 13. The second is a continuous transition in the Ising universality class. A fluctuation analysis indicates that the transitions are mean field in nature. This reflects the dominating influence of long-range Coulomb interactions between the unscreened charges that are bound to the rods. At the end we speculate on how the singularities associated with such transitions could be relevant to the physics of packaging and other bundling phenomena in biology. It is worth noting that structural transitions have been recently observed in DNA-dendrimer complexes [14]. These transitions do not appear to correspond to the specific ones we discuss. However, the notion of structural transitions in complexes of rods and localized charges clearly has an experimental as well as a theoretical basis.

We also touch on, but do not discuss in detail, the counterion "melting" transition expected when the rods are far apart. This leads to a "counterion liquid." The attraction between rods in such a state has been extensively explored by Ha and Liu, 2, 15, 16. The melting transition is continuous and can be shown to be in the universality class of the three-dimensional $X Y$ model; see, e.g., 17, 18, 19, 20]. A brief review of the arguments leading to this conclusion appears in an appendix.

An outline of this paper is as follows. The following section contains the characterization of the lattice and 
the Coulomb energy calculations. Section [III addresses "melting" of the counterion lattice, while Section [V]contains concluding remarks. A series of appendices address some technical issues. Appendix A contains a calculation of the Coulomb energy of a lattice of infinite, uniformly charged rods. Appendix B provides the derivation of the term in the energy quadratic in displacements of counterions from their lattice sites (i.e., harmonic "phonon" dispersion relation). Appendix C provides some heuristics on the "melting" of the counterion lattice.

\section{LATTICE OF RODS AND COUNTERION ENERGY}

The "lattice" consisting of a set of negatively charged rods and attached polyvalent counterions of charge $+Z e$ will be treated as a simple ionic lattice. At the first stage of approximation, the fixed charge of the rods will be replaced by a uniform negatively charged background (as in the jellium model of interacting electrons 21]). The counterions will be assumed to form a three-dimensional lattice that generalizes the close-packing arrangement in a face-centered cubic (fcc) crystal. Given that the rods are infinitesimally thin, there is no prospect of a two-dimensional arrangement of charges on the surface of any of them [3]. As has been noted in the literature [22], such a lattice can be constructed starting from a two-dimensional hexagonal close-packed structure, via the introduction of three sublattices. Our generalization makes use of this construction. No significant error is introduced by our neglect of the explicit contribution of the rods themselves to electrostatic interactions. Dimensional considerations and detailed calculations (see Appendix $\mathrm{A}$ ) lead to the conclusion that the Coulomb energy due to interactions between charged rods consists of two contributions, the first independent of the separation between rods and the second going as the logarithm of the separation between rods, assuming overall charge neutrality. Given that the counterions are forced to sit on the rods, the principal outcome of the rod-counterion interaction arises from the overall charge neutrality enforced by the charge on the rods.

\section{A. Structure of the rod-counterion lattice}

Imagine a large bundle of hexagonally close-packed pencils or rods. Viewed end-on, the rods lie on three triangular sublattices, as depicted in Fig. 1 Now pass planes at regular spacing $l_{v}$ perpendicular (initially) to the rods. Where the first plane intersects the rods on the first sublattice, place counterion charges. Likewise treat the second and third planes at their intersections with rods on the second and third sublattices, respectively. Repeat this pattern with subsequent planes, thereby building an infinite three-dimensional modified fcc lattice. Figure 2 displays the lattice from a viewpoint per-

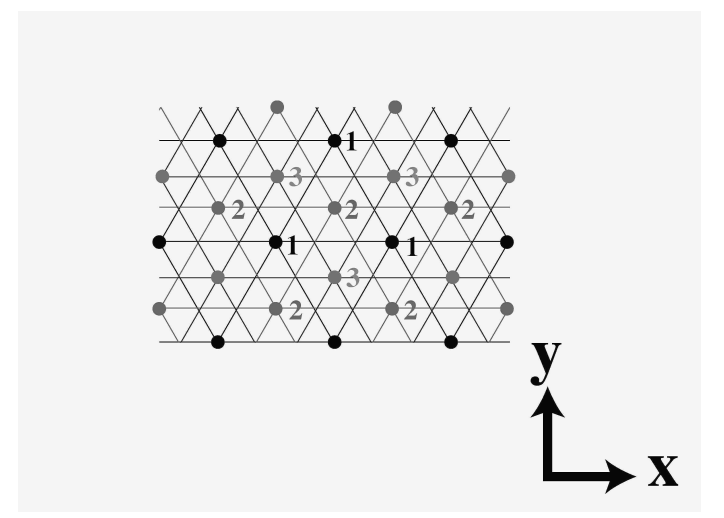

FIG. 1: Schematic of the counterion lattice, seen end-on with respect to the rods that support it. The rods are hexagonally close-packed, and the three sublattices occupied by the counterions are indicated.

pendicular to the axis of the rods. The three counterion sublattices are evident if one scans the figure from left to right. If $l_{v}$ is adjusted appropriately relative to the lattice constant of one triangular sublattice, $l_{h}$, the counterion charges themselves sit on a true fcc lattice.

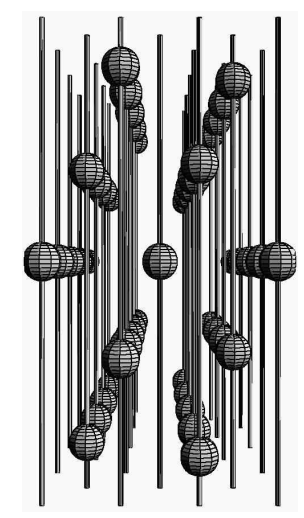

FIG. 2: A view of the lattice perpendicular to the axis of the rods. The spheres represent the counterions. Scanning from left to right, one observes the three sublattices.

The three primitive vectors of the Bravais lattice of counterions so constructed are

$$
\begin{aligned}
& \vec{a}_{1}=\frac{1}{2} l_{h} \hat{x}+\frac{\sqrt{3}}{2} l_{h} \hat{y}+l_{v}\left(g_{x}+g_{y} \sqrt{3}\right) \hat{z} \\
& \vec{a}_{2}=-\frac{1}{2} l_{h} \hat{x}+\frac{\sqrt{3}}{2} l_{h} \hat{y}+l_{v}\left(-g_{x}+g_{y} \sqrt{3}\right) \hat{z} \\
& \vec{a}_{3}=\frac{1}{\sqrt{3}} l_{h} \hat{y}+l_{v}\left(1+\frac{2}{\sqrt{3}} g_{y}\right) \hat{z}
\end{aligned}
$$

The dimensionless vector $\vec{g}=\hat{x} g_{x}+\hat{y} g_{y}$ encodes the possibility of tilting the planes of counterions. The corresponding primitive vectors of the reciprocal lattice, $\vec{b}_{i}$, are constructed in the standard way 22], so that the relationship between the two sets of primitive vectors is $\vec{a}_{i} \cdot \vec{b}_{j}=2 \pi \delta_{i, j}$. To recover the true fcc lattice, the aspect 
ratio, $r \equiv l_{h} / l_{v}$, is taken to be $\sqrt{3 / 2}$ and $\vec{g}$ is set equal to zero. The aspect ratio will play the role of a control parameter in what follows.

Assume overall charge neutrality of the rod-counterion system, so that there is exact cancellation between the mean charge per unit volume of the counterion lattice and the uniformly charged negative background provided by the rods. We make use of the Ewald method for the evaluation of the Coulomb sum 21, 22, 23]. The "reduced" Coulomb energy is defined as

$$
\begin{aligned}
& E_{\text {Coul }} /\left(Z^{2} e^{2} / 2 l_{v}\right) \\
& \equiv \tilde{E}_{\text {Coul }} \\
& =\sum_{\vec{n} \neq 0} \frac{l_{v}}{\left|n_{1} \vec{a}_{1}+n_{2} \vec{a}_{2}+n_{3} \vec{a}_{3}\right|}-S
\end{aligned}
$$

where $\vec{n}$ is a triplet of integers $\left(n_{1}, n_{2}, n_{3}\right)$, and the subtraction $S$ represents the compensating interaction with the smeared out negatively charged background.

In our calculation, we assume that the distance between "planes" of ions is fixed at $l_{v}$. The effect of a compression of the lattice of polyelectrolytic rods is to decrease the spacing, $l_{h}$.

\section{B. The Coulomb sum}

Using Ewald summation techniques 21, 22, 23], one generates an expression for the Coulomb energy that can be expressed in terms of the sum of four terms. Those terms are

$$
-\frac{2}{\sqrt{\pi}} \alpha^{1 / 2}\left(l_{h}^{2} l_{v}\right)^{-1 / 3}
$$

$$
\begin{aligned}
& \sum_{\vec{n} \neq 0} \frac{1}{\left|n_{1} \vec{a}_{1}+n_{2} \vec{a}_{2}+n_{3} \vec{a}_{3}\right|} \\
& \quad \times\left(1-\operatorname{erf}\left(\left|n_{1} \vec{a}_{1}+n_{2} \vec{a}_{2}+n_{3} \vec{a}_{3}\right| \alpha^{1 / 2}\left(l_{h}^{2} l_{v}\right)^{-1 / 3}\right)\right)
\end{aligned}
$$

$$
\begin{gathered}
-\frac{\pi}{v} \alpha^{-1}\left(l_{h}^{2} l_{v}\right)^{2 / 3} \\
\frac{4 \pi}{v} \sum_{\vec{m} \neq 0} \frac{e^{-\left|m_{1} \vec{b}_{1}+m_{2} \vec{b}_{2}+m_{3} \vec{b}_{3}\right|^{2} \alpha^{-1}\left(l_{h}^{2} l_{v}\right)^{2 / 3} / 4}}{\left|m_{1} \vec{b}_{1}+m_{2} \vec{b}_{2}+m_{3} \vec{b}_{3}\right|^{2}}
\end{gathered}
$$

The quantity $\alpha$ in the expressions above is an adjustable parameter, which is ideally set equal to a value that maximizes convergence of the Ewald sums in (2.6) and (2.8). A close-to-optimal choice is $\alpha=4 \pi$.

The mean-field phase diagram of the lattice can be determined by examining the dependence of the Coulomb energy on the aspect ratio, $r$, and the tilt vector, $\vec{g}$. What one finds is that for $r$ greater than a threshold value $r_{a} \approx$
1.1 the Coulomb energy is minimized when $\vec{g}=0$. At this threshold value, three minima lying symmetrically in the $\vec{g}$-plane represent equally low energies. This situation is illustrated in Figure 3] When $r<r_{a}, \vec{g}=0$ no longer

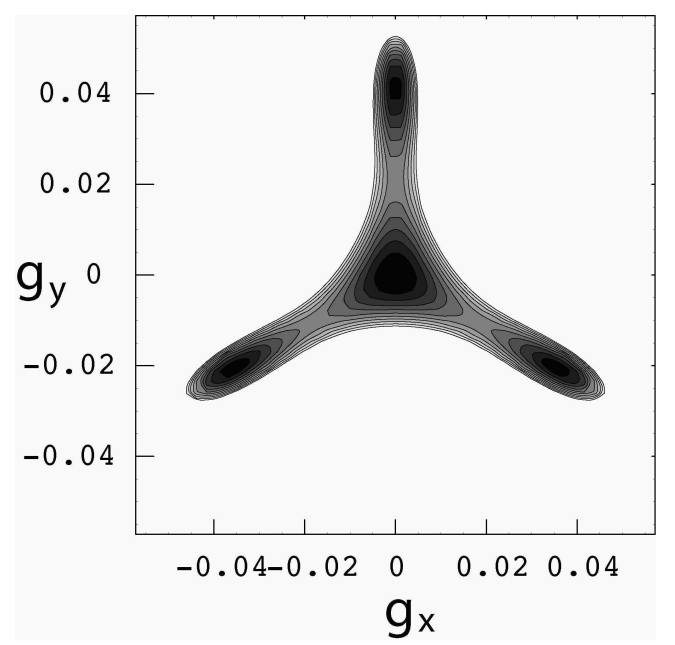

FIG. 3: Contour plot of the Coulomb energy of the generalized fcc counterion lattice in the $g_{x}-g_{y}$ plane at $r=r_{a} \approx 1.1$. The four minima, including the one at $\vec{g}=0$, are all of equal depth.

represents a global minimum of the energy. At $r=r_{s} \approx$ 1.097, the local minimum at $\vec{g}=0$ disappears. In this sense, one can think of $r_{s}$ as a "spinodal" point. Note the small difference between $r_{s}$ and $r_{a}$. The transition at $r_{a}$ is weakly first order.

As $r$ is further reduced, corresponding to even closer packing of the rods, a new structural transition is encountered, at which the three minima each split into two new ones. The aspect ratio, $r_{b}$, at which this transition takes place is approximately equal to 0.801. Contour plots illustrating the onset of this transition and the evolution of the new minimum energy configurations are shown in Figure 4. That this transition is also a symmetry-breaking one is evident from Fig. 4. Here, the transition is continuous, in that the appearance of the six minima at lower aspect ratio is simultaneous with the disappearance of the three minima associated with values of $r$ greater than $r_{b}$. At mean field level this transition is of the standard second-order type. Given the nature of the symmetry-breaking, it is altogether reasonable to classify it as an Ising-like, or $O(1)$, phase transition. In the case at hand, the aspect ratio plays the role of temperature. This means that singularities that one expects to find in the entropy and specific heat of a thermal system will show up here in the form of nonanalyticities in the dependence of the energy as a function of spacing between rods, with direct consequences on packing forces.

In light of the continuous nature of the phase transition at $r=r_{b}$ and the weakness of the first-order phase transition at $r=r_{a}$, the question of the effects of fluctuations is clearly relevant. We address this question with the use of coarse-grained effective Hamilitonians. Assume that 

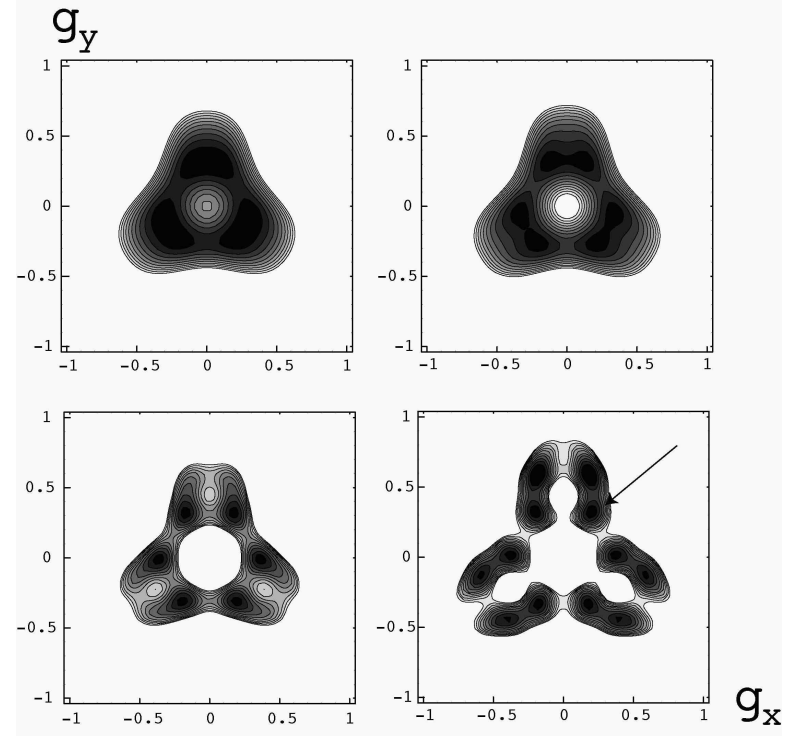

FIG. 4: The emergence of six minima in the Coulomb energy, and the migration of those minima as the aspect ratio is decreased below the threshold value $r_{b}=0.801$. Reading from left to right and top to bottom, the values of $r$ are equal to $0.801,0.693,0.577$ and 0.433 , respectively. The arrow in the bottom-right-hand figure points to one of the six true minima of the energy.

the counterions are only allowed to move along the rods. Then, at the onset of the first-order transition, we can express the fluctuations in the locations of the counterions in terms of a scalar displacement field, $u(\vec{r})$ that quantifies counterions displacments paralles to the rods. The Coulomb energy can then be written as an expansion in terms of $u$. This energy is most usefully expressed in terms of the Fourier transform of the displacement field. The most relevant terms in the expansion yield the expression

$$
\begin{aligned}
H= & \sum_{\vec{k}, Q}\left[|\vec{k}|^{2}\left(r-r_{s}\right)+C k^{4}+A Q^{2}+B \frac{Q^{2}}{k^{2}+Q^{2}}\right] \\
& \times u(\vec{k}, Q) u(-\vec{k},-Q) \\
& +\sum_{\vec{q}_{1}, \ldots, \vec{q}_{3}} w_{3}\left(\vec{q}_{1}, \vec{q}_{2}, \vec{q}_{3}\right) u\left(\vec{q}_{1}\right) u\left(\vec{q}_{2}\right) u\left(\vec{q}_{3}\right) \delta_{\vec{q}_{1}+\vec{q}_{2}+\vec{q}_{3}} \\
& +\sum_{\vec{q}_{1}, \ldots, \vec{q}_{4}} w_{4}\left(\vec{q}_{1}, \vec{q}_{2}, \vec{q}_{3}, \vec{q}_{4}\right) u\left(\vec{q}_{1}\right) u\left(\vec{q}_{2}\right) u\left(\vec{q}_{3}\right) u\left(\vec{q}_{3}\right) \\
& \times \delta_{\vec{q}_{1}+\vec{q}_{2}+\vec{q}_{3}+\vec{q}_{4}}
\end{aligned}
$$

Here, we have split the three-dimensional wave vector $\vec{q}$ into a two-dimensional vector, $\vec{k}$ in the $x-y$ plane and a component, $Q$, in the $z$-direction. The term with coefficient $B$ is the contribution to the quadratic energy reflecting the long-range nature of the unscreened Coulomb interactions between counterions. We have inserted the "stabilizing" quartic term going as $k^{4}$, but have ignored the inessential term proportional to $Q^{4}$, or the cross term, proportional to $k^{2} Q^{2}$. It is important to keep in mind that the actual stabilization of the system results from the shearing transition.

The higher order terms in the the effective Hamiltonian (2.9) have the following forms:

$$
w_{3}\left(\vec{q}_{1}, \vec{q}_{2}, \vec{q}_{3}\right)=-C_{1}\left(k_{y}^{3}-3 k_{y} k_{x}^{2}\right)
$$

and

$$
\begin{aligned}
& w_{4}\left(\vec{q}_{1}, \cdots, \vec{q}_{4}\right) \\
&= W\left(\vec{q}_{1}, \cdots, \vec{q}_{4}\right) \\
&+B_{1}\left[\left(\vec{k}_{1} \cdot \vec{k}_{2}\right)\left(\vec{k}_{3} \cdot \vec{k}_{4}\right)+\text { permutations }\right] \\
&+B_{2} Q_{1} Q_{2} Q_{3} Q_{4} \\
&+B_{3}\left[\left(\vec{k}_{1} \cdot \vec{k}_{2}\right) Q_{3} Q_{4}+\text { permutations }\right] .
\end{aligned}
$$

Here,

$$
\begin{aligned}
& W\left(\vec{q}_{1}, \cdots, \vec{q}_{4}\right) \\
& =v\left(\vec{q}_{1}+\vec{q}_{2}\right)+v\left(\vec{q}_{1}+\vec{q}_{3}\right)+v\left(\vec{q}_{2}+\vec{q}_{3}\right) \\
& \quad-v\left(\vec{q}_{1}\right)-v\left(\overrightarrow{q_{2}}\right)-v\left(\vec{q}_{3}\right)-v\left(-\vec{q}_{1}-\vec{q}_{2}-\vec{q}_{3}\right)
\end{aligned}
$$

where

$$
v(\vec{q})=\frac{Q^{4}}{|\vec{q}|^{2}} .
$$

There are three distinct permuations of the indices in the first line of the right-hand-side of Eq. 2.11 and six distinct permutations in the last line of the right-hand-side of that equation. The term $W\left(\vec{q}_{1}, \ldots, \vec{q}_{4}\right)$ appears to be the most relevant contribution to the fourth-order coupling in the Ginzburg-Landau-Wilson model appropriate to this system. However, as it turns out, the most important term in Eq. 2.11 is the first one in the square brackets, going as $k^{4}$.

That the contributions to the energy associated with fluctuations about the lattice have the forms shown above can be established through explicit evaluation of Coulomb-type lattice sums. Appendix [B] outlines the calculation in the case of of the quadratic terms and presents results for that and the fourth order term that are obtained through explicit evaluation of those sums.

To perform an analysis of the effects of fluctuations on this weak first-order transition, one can consider a "Ginzburg criterion" 24] applied to the one-loop contribution to the "entropy" of the system 25]. Recall that the leading contribution to the mean field entropy goes as $r-r_{s}$ for $r \rightarrow r_{s}+$. Given the form of the of the quadratic term in Eq. (2.9), and recalling that $s \sim \partial F / \partial r$, with $F$ the free energy, we have the following expression for the one-loop entropy,

$$
\begin{aligned}
s & \sim \int \frac{k^{2} d^{3} q}{\left(r-r_{s}\right) k^{2}+C k^{4}+A Q^{2}+B \frac{Q^{2}}{k^{2}+Q^{2}}} \\
& \rightarrow \int \frac{k^{2} d^{2} k d Q}{\left(r-r_{s}\right) k^{2}+C k^{4}+B \frac{Q^{2}}{k^{2}}} .
\end{aligned}
$$


The expression on the right-hand-side of Eq. (2.14) contains the important terms in the denominator. A variety of methods exist for the evaluation of the integrals in this expression. The essence of the results follows from a rescaling $Q=k^{2} x$. Then, the integral to perform is

$$
\int \frac{k^{2} d^{2} k d x}{\left(r-r_{s}\right)+C k^{2}+B x^{2}}
$$

We now note that this has the same qualitative dependence on the "reduced temperature," $r-r_{s}$, as the corresponding one-loop integral of a five-dimensional $O(n)$ model with short range interactions. A further rescaling of the integration variables produces a leading singularity proportional to $\left(r-r_{s}\right)^{3 / 2}$ compared to the mean-field result $\propto r-r_{s}$. This application of the Ginzburg criterion shows that the transition is unrenormalized. A calculation of the renormalization of the fourth-order interaction leads to the same conclusion, namely that fluctuations lead to a well-behaved change in the amplitude. This leads to the conclusion that, because of the long range dipole-dipole interactions between the charges embedded on the rods, the first-order shearing phase transition of the charge lattice at $r=r_{a}$ is essentially mean field in nature. Similar arguments, allowing for modifications in the form of Eq. (2.9) by, for example, breaking rotational symmetry in the $x-y$ plane, reveal that fluctuations don't modify the mean-field continuous shearing transition. Technically, the transition at $r=r_{b}$ is Ising-like with effective dimensionality $d>4$.

The full results of the Coulomb energy calculations are shown in Fig. [5] The analysis above reveals that fluctuations don't qualitatively change the picture.

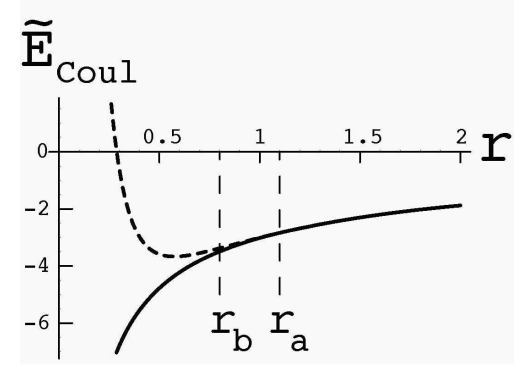

FIG. 5: Solid line: the reduced Coulomb energy as a function of aspect ratio in the vicinity of the first-order and continuous transitions. Dashed line: the energy if the counterion lattice is constrained not to tilt. The locations of the transitions are indicated on the figure.

Figure 6 displays the (very small) discontinuity in the derivative $\partial \tilde{E}_{\text {Coul }} / \partial r$ at the first order transition at $r=$ $r_{a}$.

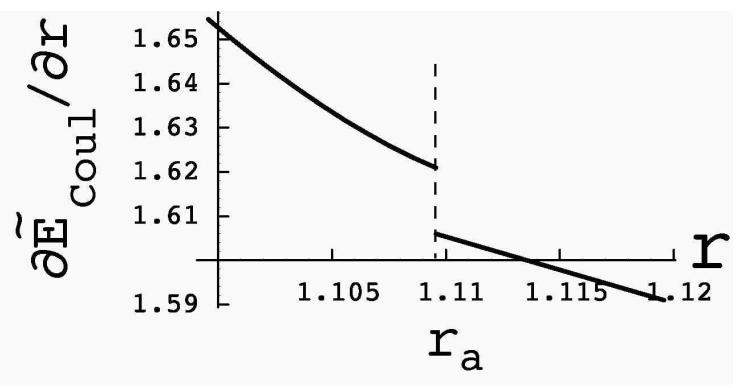

FIG. 6: A plot of $\partial \tilde{E}_{\text {Coul }} / \partial r$ in the immediate vicinity of $r=r_{a}$, illustrating the discontinuity there, associated with the first order phase transition to the sheared lattice.

\section{III. "MELTING" OF THE COUNTERION LATTICE}

The three-dimensional lattice of statically correlated counterions being discussed can be reasonably expected to exist at low enough temperatures and for sufficiently close packing of the lattice of rods. At high temperatures and when the spacing between rods is relatively large, the counterion lattice will not be stable against thermal fluctuations. A variety of arguments lead to criteria for the existence of this lattice. The simplest compares the (negative) interaction energy of the lattice to $k_{B} T$. It is reasonable to expect that the lattice will resist the disordering effects of thermal fluctuations when

$$
\left|\frac{Z^{2} e^{2}}{2 l_{v}} \tilde{E}_{\text {Coul }}\right| \geq k_{B} T
$$

Assuming that the general condition, $Z^{2} e^{2} /\left(k_{B} T l_{v}\right) \gg 1$ for the existence of a "Wigner crystal" in the couterionpolyelectrolyte system are met [3], we expect the crystal discussed here to be stable as long as $\left|\tilde{E}_{\text {Coul }}\right|$ is of order unity or greater. According to Fig. [5 this should be the case as for a range of aspect ratios that encompass the two structural transitions on which this paper has focussed.

An alternative estimation of the threshold at which melting of the counterion lattice takes place is based on the Lindemann criterion 21], according to which a lattice melts when thermally-induced displacements are some fraction of the lattice spacing. This leads to approximate predictions for the melting transition that we expect to be consistent with the energy-vs.-entropy arguments leading to the criterion (3.1) for the stability of the counterion lattice against melting.

When melting takes place, it does so in the same way that charge density waves disappear, that is to say, continuously, with thermodynamic signatures that identify its universality class as that of the $3 D X Y$ model. Theoretical arguments and experimental observations that justify this conclusion in the case of charge density wave systems can be found in the literature [17, 18, 19, 20]. Appendix [C] contains a brief, heuristic argument for the nature of the melting transition in the counterion system. 


\section{CONCLUSIONS}

As noted above, the singularities in the dependence of the Coulomb energy on aspect ratio have implications with respect to the energetics of bundling or packaging polyelectrolytic molecules. These implications follow from the effective force exerted by the decorated rods on each other, as determined by the "pressure", $p \equiv-\partial E_{\text {Coul }} / \partial r$. The pressure (analogous to the entropy in a thermal system as discussed) has a discontinuity at $r=r_{a}$, and a discontinuous derivative at $r=r_{b}$. The singularities are not striking (recall Fig. 6). The kinetics of bundling or packaging such molecules with crystalized counterions are presumably affected by the Coulomb energy, and should, in principle, reflect these singuarities. What is more important, however, is the possibility that a sequence of structural transitions in the counterion lattice assists compression to high densities. In Fig. 5] we have shown the Coulomb energy computed directly from the Ewald sums in the vicinity of the firstorder transition at $r=r_{a}$ and the continuous transition at $r=r_{b}$. The dashed curve shows the energy if the lattice were constrained not to tilt. It is interesting to contemplate whether in some instances Nature relieves the strong Coulomb repulsion via structural transitions.

It is important to note that one cannot argue for structural transitions as the sine qua non of polyelectrolyte packaging. Specifically, in the packaging of DNA in tailtype bacteriophages, ATP is known to provide the fuel for a packaging motor [8, 26]. Order of magnitude estimates suggest that $\sim 50-60 p N$ forces generated by this motor [26] suffice to overcome Coulomb forces and compress a rod-like system to observed densities without benefit of structural transitions. Of course, in experimental situations other repulsive energies besides Coulomb are also involved. 27, 28]

The present analysis does not suggest dramatic consequences in actin bundling, or in the kinetics of packaging and/or infection in a bacteriophage life-cycle. However, a continuous shearing transition, if realized, should be accompanied by strong counterion charge fluctuations, which could be susceptible to dynamic scattering experiments. The tilting, estimated to be a few percent at the first-order transition, could potentially be detected by standard diffraction techniques.

While, strictly speaking, we have shown that a shearing transition ought to occur in a sufficiently closelypacked, constrained polyelectrolytic system, we have not ruled out the possibility that some other transition intercedes, preempting this particular rearrangement as the system of rods is compressed. One would have to consider possibilities for ever larger unit cells in the rod lattice. More importantly, we can only suggest the possibility that tilting or other structural transition(s) occur as biologically relevant polyelectrolytic molecules condense. We hope this work will stimulate further experiments on, for example, the kinetics of DNA packaging, since such a transition could occur "on the fly" as the "spooling" progresses. 28, 29] In other potential experiments, DNA or other bundles of varying density could be prepared and probed statically.

We thank Prof. A. B. Harris, Dr. Ron Fisch and Prof. Roger Hendrix for helpful comments. We are also grateful to Prof. Robijn Bruinsma for bringing Ref. [14] and the work on which it reports to our attention.
[1] F. Oosawa, Polyelectrolytes (M. Dekker, New York,, 1971).

[2] B. Y. Ha and A. J. Liu, Phys. Rev. Lett. 79, 1289 (1997).

[3] B. I. Shklovskii, Phys. Rev. Lett. 82, 3268 (1999).

[4] N. Gronbech-Jensen, R. J. Mashl, R. F. Bruinsma, and W. M. Gelbart, Phys. Rev. Lett. 78, 2477 (1997).

[5] F. Oosawa, Biopolymers 6, 134 (1968).

[6] T. Ohnishi, N. Imai, and F. Oosawa, J. Phys. Soc. Jpn 15, 896 (1960).

[7] V. Bloomfield, Biopolymers 31, 1471 (1991).

[8] R. W. Hendrix, Cell 94, 147 (1998).

[9] J. D. Watson, Molecular biology of the gene (Benjamin/Cummings, Menlo Park, 1988), 4th ed.

[10] D. Durand, J. Doucet, and F. Livolant, Journal de Physique II (Atomic, Molecular and Cluster Physics, Chemical Physics, Mechanics and Hydrodynamics) 2, 1769 (1992).

[11] J. Pelta, D. Durand, J. Doucet, and F. Livolant, Biophysical Journal 71, 48 (1996).

[12] T. E. Angelini, H. Liang, W. Wriggers, and G. C. Wong, Proc Natl Acad Sci U S A (2003).

[13] D. J. Amit and A. Shcherbakov, J. Phys. C (Solid State) 7, L96 (1974).
[14] H. M. Evans, A. Ahmad, K. Ewert, T. Pfohl, A. MartinHerranz, R. F. Bruinsma, and C. R. Safinya, Structural polymorphism of DNA-dendrimer complexes, unpublished.

[15] B. Y. Ha and A. J. Liu, Physical Review E (Statistical Physics, Plasmas, Fluids, and Related Interdisciplinary Topics) 58, 6281 (1998).

[16] B. Y. Ha and A. J. Liu, Physical Review E (Statistical Physics, Plasmas, Fluids, and Related Interdisciplinary Topics) 60, 803 (1999).

[17] G. Grüner, Density waves in solids, Frontiers in physics ; v. 89. (Addison-Wesley, Reading, 1994).

[18] S. Girault, A. H. Moudden, and J. P. Pouget, Phys. Rev. B 39, 4430 (1989).

[19] H. Fukuyama and H. Takayama, in Electronic properties of inorganic quasi-one-dimensional compounds. Part I: Theoretical, edited by P. Monceau (D. Reidel ; Sold and distributed in the U.S.A. and Canada by Kluwer Academic Publishers, Dordrecht Holland ; Boston Hingham, MA, U.S.A., 1985), pp. 41-104.

[20] J. W. Brill, M. Chung, Y. K. Kuo, X. Zhan, E. Figueroa, and G. Mozurkewich, Physical Review Letters 74, 1182 (1995). 
[21] J. M. Ziman, Principles of the theory of solids (Cambridge U. P., New York, 1979), 1st ed.

[22] N. W. Ashcroft and N. D. Mermin, Solid state physics (Holt, New York, 1976).

[23] P. P. Ewald, Ann. Physik 64, 253 (1921).

[24] V. L. Ginzburg, Sov. Phys. Solid State 2, 1824 (1961).

[25] Y. M. Ivanchenko and A. A. Lisyansky, Physics of critical fluctuations, Graduate texts in contemporary physics (Springer-Verlag, New York, 1995).

[26] D. E. Smith, S. J. Tans, S. B. Smith, S. Grimes, D. L. Anderson, and C. Bustamante, Nature 413, 748 (2001).

[27] S. C. Riemer and V. Bloomfield, Biopolymers 17, 785 (1978).

[28] T. Odijk, Biophys J 75, 1223 (1998).

[29] L. W. Black, in The Bacteriophages, edited by R. Calender (Plenum, New York, 1988), vol. 2, pp. 321-373.

\section{APPENDIX A: THE COULOMB SUM FOR UNIFORMLY CHARGED RODS}

As discussed in the text, the charges on the rods have been assumed to be smeared out into a three-dimensional smooth background, analogous to the jellium model used in discussions of electron-electron interactions in a metal 21]. In this Appendix, we evaluate the corrections to this approximation by now assuming that the backbone charges are uniform along the rods. The method utilized to evaluate the alteration in the Coulomb energy that is induced by this refinement in the model is a version of the Ewald summation technique [21, 22, 23]. The quantity we will calculate is the potential energy of a charged test rod in the presence of an array of uniformly charged rods, which are assumed to be in a hexagonal close-packed arrangement. At the end, the test rod will be moved onto a rod of the lattice. Before doing this, we eliminate ("subtract") the interaction between the test rod charge and the rod on which it eventually sits.

Because the rods are uniformly charged in the $z$ direction, the potential at the two-dimensional location $\vec{r}$ due to a rod at the origin is given by

$$
\phi(\vec{r}) \propto \frac{1}{\pi} \int \frac{e^{i \vec{q} \cdot \vec{r}}}{q^{2}} d^{2} q=\frac{1}{\pi} \int_{0}^{\infty} d t\left\{\int e^{i \vec{q} \cdot \vec{r}-q^{2} t} d^{2} q\right\}
$$

We split the $t$-integration into one from 0 to $T$ and another from $T$ to $\infty$, where $T$ is, initially, arbitrary. We suppose that the distance between nearest-neighbor rods in the close-packed lattice is $l_{h}$. Given this, we will take $T \propto l_{h}^{2}$. The primitive vectors for this lattice can be taken to be

$$
\begin{aligned}
\vec{a}_{1} & =l_{h} \hat{x} \\
\vec{a}_{2} & =\frac{l_{h}}{2} \hat{x}+\frac{\sqrt{3} l_{h}}{2} \hat{y},
\end{aligned}
$$

and the volume of the primitive cell for this lattice is

$$
v_{W S}=\frac{\sqrt{3}}{2} l_{h}^{2}
$$

The corresponding reciprocal lattice vectors are

$$
\begin{aligned}
& b_{1}=\frac{2 \pi}{l_{h}}\left(\hat{x}-\frac{1}{\sqrt{3}} \hat{y}\right) \\
& b_{2}=\frac{4 \pi}{\sqrt{3} l_{h}} \hat{y},
\end{aligned}
$$

while the volume of the primitive cell of the reciprical lattice is given by

$$
v_{B Z}=\frac{8 \pi^{2}}{\sqrt{3} l_{h}^{2}} .
$$

For the integration from $t=T$ to $t=\infty$, we make use of the two-dimensional version of the Poisson sum formula:

$$
\sum_{\vec{r}=\vec{R}_{i}} f(\vec{r})=\frac{1}{v_{W S}} \sum_{\vec{Q}_{k}} \int e^{i \vec{Q}_{k} \cdot \vec{r}} f(\vec{r}) d^{2} r,
$$

where $\left\{\vec{R}_{i}\right\}$ correspond to a hexagonal lattice, and where the $\vec{Q}_{k}$ range over the reciprocal lattice. We then have for the contribution of this region of $t$-integration to the potential energy of a point charge at the location $\vec{r}$

$$
\begin{aligned}
& \frac{1}{\pi v_{W S}} \sum_{\vec{Q}_{k}} \int d^{2} r \int d^{2} q e^{i\left(\vec{q}+\vec{Q}_{k}\right) \cdot r} \frac{e^{-T q^{2}}}{q^{2}} \\
& =\frac{4 \pi}{v_{W S}} \sum_{\vec{Q}_{k}} \frac{e^{-T Q_{k}^{2}} e^{i \vec{Q}_{k} \cdot \vec{r}}}{Q_{k}^{2}} .
\end{aligned}
$$

The only tricky part here is the term for which $\vec{Q}_{k}=0$, corresponding to the smeared-out portion of the distribution. This can be handled by a careful subtraction. Because of the fact that $T \propto l_{h}^{2}$ and the fact that the $\vec{Q}_{k}$ 's go as $l_{h}^{-2}$, terms in (A9) are independent of the spacing between rods, $l_{h}$.

The next portion of the integration is from 0 to $T$. Here, we sum directly in real space. We find

$$
\begin{aligned}
& \frac{1}{\pi} \sum_{\vec{R}_{l}} \int_{h}^{T} d t \int d^{2} q e^{i \vec{q} \cdot\left(\vec{r}-\vec{R}_{l}\right)} e^{-t q^{2}} \\
& =\sum_{\vec{R}_{l}} \int_{1}^{\infty} e^{-\left|\vec{r}-\vec{R}_{l}\right|^{2} t / 4 T} \frac{1}{t} d t .
\end{aligned}
$$

A careful subtraction eliminates the divergent contribution of term in the sum in which $\vec{R}_{l}=\vec{r}$. The divergence arises when the limit $\vec{r} \rightarrow 0$ is taken, corresponding to placing the test rod on one of the rods in the lattice. One subtracts the standard energy associated with the Coulomb interaction between the test rod charge and the rod at the origin. This subtraction leads to the only term with any $l_{h}$ dependence, going as $\ln l_{h}$. All other dependence disappears because of cancellations between the $l_{h}$-dependence of the $\vec{Q}_{k}$ 's, the $\vec{R}_{l}$ 's and $T$.

The subtraction in A9 associated with the $\vec{Q}_{k}=0$ term is easily carried out, as is the subtraction in the case 
of the term associated with a test rod position $\vec{r}$ directly on a rod, in A10. Choosing specifically

$$
T=\frac{\alpha l_{h}^{2}}{4 \pi}
$$

with $\alpha$ an arbitrary constant, one can perform the sums indicated above, with the appropriate subtractions. One finds the following result for the Coulomb sum:

$$
-2.78608+2 \ln l_{h}
$$

Note that the above result must be independent of the arbitrary constant $\alpha$. We are now in a position to include more precisely the effect of the concentration of negative charge on the polyelectrolyte rods. One must multiply the result A12 by

$$
\sigma^{2}=\left(\frac{Z e}{l_{v}}\right)^{2}
$$

where $\sigma$ is the linear charge density on the rods, $Z$ is the valence of the condensed counterions, and $l_{v}$ is the spacing between counterions on the rod. The above multiplication will yield the energy of the rod lattice is per unit rod length.

We are thus led to an expression for the contribution of the rod-rod interaction to the Coulomb energy; it has a non-trivial, but smooth dependence on the separation between rods. This energy adds as a "background" term to the energies calculated in the body of this paper. A very similar calculation is used to compute the interaction energy between the counterion lattice and the uniformly charged rod lattice. Both contributions have no effect on the structural transitions discussed in the body.

\section{APPENDIX B: EWALD SUM FOR THE SECOND AND HIGHER ORDER TERMS IN COUNTERION DISPLACEMENTS}

The result for the energy cost of a distortion of the counterion lattice follows straightforwardly from the expression for the energy of a collection of interacting charges. We start by writing the two-particle interaction energy in terms of its spatial Fourier transform,

$$
V(\vec{r})=\int d^{3} q v(\vec{q}) e^{i \vec{q} \cdot \vec{r}}
$$

Then the positions of the counterions, $\vec{r}_{j}$, are expanded in terms of displacements from the lattice sites, $\vec{u}\left(\vec{R}_{j}\right)$. Assuming that those displacements are entirely in the $z$ direction, in line with our model in which the counterions are bound to the charged polyelectrolye rods, the displacment of the $j^{\text {th }}$ counterion from its equilibrium position on the counterion lattice will be equal to $\hat{z} u\left(\vec{R}_{j}\right)=u_{j} \hat{z}$. Expanding to second order in the $u$ 's, we obtain for the energy associated with those distortions

$$
\sum_{\vec{q}}\left[\sum_{\vec{Q}}\left(\left(q_{z}+Q_{z}\right) v(\vec{q}+\vec{Q})-Q_{z} v(\vec{Q})\right)\right] u(\vec{q}) u(-\vec{q}),
$$

where $u(\vec{q})$ is the spatial Fourier transform of the lattice displacement $u_{j}$ :

$$
u(\vec{q})=\sum_{j} u_{j} e^{-i \vec{q} \cdot \vec{R}_{j}}
$$

Given that the interactions are Coulomb, the sum of interest is of the form

$$
\sum_{\vec{Q}} \frac{\left(q_{z}+Q_{z}\right)^{2}}{|\vec{Q}+\vec{q}|^{2}}-\sum_{\vec{Q}} \frac{\left(Q_{z}\right)^{2}}{|\vec{Q}|^{2}}
$$

where the $\vec{Q}$ 's are displacement vectors on the reciprocal lattice. The primitive displacement vectors on this lattice are $\vec{b}_{1}, \vec{b}_{2}$ and $\vec{b}_{3}$. The primitive displacement vectors on the original lattice are $\vec{a}_{1}, \vec{a}_{2}$ and $\vec{a}_{3}$. The relationship between the $\vec{b}$ 's and the $\vec{a}$ 's is

$$
\vec{b}_{1}=2 \pi \frac{\vec{a}_{2} \times \vec{a}_{3}}{\vec{a}_{1} \cdot\left(\vec{a}_{2} \times \vec{a}_{3}\right)}
$$

and similarly for $\vec{b}_{2}$ and $\vec{b}_{3}$. The primitive volume in the reciprocal lattice is the volume of the first Brillouin zone, given by

$$
v_{B Z}=\left|\vec{b}_{1} \cdot\left(\vec{b}_{2} \times \vec{b}_{3}\right)\right|
$$

The primitive volume in the real lattice is the volume of the Wigner-Seitz cell, given by

$$
v_{W S}=\left|\vec{a}_{1} \cdot\left(\vec{a}_{2} \times \vec{a}_{3}\right)\right|
$$

Given the relationship between the $\vec{a}$ 's and the $\vec{b}$ 's, the the following holds

$$
v_{B Z} v_{W S}=(2 \pi)^{3}
$$

The Poisson sum formula in three dimensions takes the following form:

$$
\sum_{\vec{Q}} f(\vec{Q})=\frac{1}{v_{B Z}} \int d^{3} Q \sum_{\vec{R}} e^{i \vec{R} \cdot \vec{Q}} f(\vec{Q})
$$

The sum on the right hand side is over all lattice points on the real space lattice. One final relationship between the $\vec{a}$ 's and the $\vec{b}$ 's is

$$
\vec{a}_{i} \cdot \vec{b}_{j}=2 \pi \delta_{i, j}
$$

In (B9) each $\vec{Q}$ is of the form $m_{1} \vec{b}_{1}+m_{2} \vec{b}_{2}+m_{3} \vec{b}_{3}$, where the $m_{i}$ 's take integral values from $-\infty$ to $\infty$. Similarly the $\vec{R}$ 's are of the form $n_{1} \vec{a}_{1}+n_{2} \vec{a}_{2}+n_{3} \vec{a}_{3}$, where the $n_{i}$ 's range over all integers, as well. 
Now, one can clearly write the expression in (B4) in the form $f(\vec{q})-f(0)$. Focus on $f(\vec{q})$ and write

$$
\frac{\left(q_{z}+Q_{z}\right)^{2}}{|\vec{q}+\vec{Q}|^{2}}=\int_{0}^{\infty}\left(q_{z}+Q_{z}\right)^{2} e^{-|\vec{q}+\vec{Q}|^{2} t} d t
$$

As in Appendix A, the integral over $t$ splits into an integral from 0 to $T$ and from $T$ to $\infty$, where, in this case, we choose

$$
\begin{aligned}
T & =\beta \frac{\pi}{\left(v_{B Z}\right)^{2 / 3}} \\
& =\beta \frac{\left(v_{W S}\right)^{2 / 3}}{4 \pi}
\end{aligned}
$$

where $\beta$ is arbitrary. For the integral from $T$ to $\infty$, one finds

$$
\begin{aligned}
\sum_{\vec{Q}} & \frac{\left(q_{z}+Q_{z}\right)^{2}}{|\vec{q}+\vec{Q}|^{2}} e^{-\pi \beta|\vec{q}+\vec{Q}|^{2} / v_{B Z}^{2 / 3}} \\
= & \frac{q_{z}^{2}}{|\vec{q}|^{2}} e^{-\beta \pi|\vec{q}|^{2} / v_{B Z}^{2 / 3}} \\
& \quad+\sum_{\vec{Q} \neq 0} \frac{\left(q_{z}+Q_{z}\right)^{2}}{|\vec{q}+\vec{Q}|^{2}} e^{-\pi \beta|\vec{q}+\vec{Q}|^{2} / v_{B Z}^{2 / 3}}
\end{aligned}
$$

The Poisson sum formula is applied to the integration over $t$ from 0 to $T$. This leads to the following expression

$$
\int d^{3} Q\left\{\frac{1}{v_{B Z}} \sum_{\vec{R}} \int_{0}^{\beta \pi / v_{B Z}^{2 / 3}=\beta v_{W S}^{2 / 3} / 4 \pi} e^{i \vec{Q} \cdot \vec{R}-|\vec{q}+\vec{Q}|^{2} t}\left(q_{z}+Q_{z}\right)^{2} d t\right\}
$$

The integral in brackets is evaluated by introducing the generating function

$$
\frac{1}{v_{B Z}} \sum_{\vec{R}} \int_{0}^{\beta v_{W S}^{2 / 3} / 4 \pi} e^{i \vec{Q} \cdot \vec{R}-|\vec{q}+\vec{Q}|^{2} t+\kappa \hat{z} \cdot(\vec{q}+\vec{Q})} d t .
$$

One obtains B14 from (B15) by taking the second derivative with respect to $\kappa$, and then setting $\kappa=0$. For non-zero $\vec{R}$, the integral over $\vec{Q}$ is taken easily enough. Completing squares, one is left with

$$
\frac{1}{v_{B Z}} \int_{0}^{\beta v_{W S}^{2 / 3} / 4 \pi}\left(\frac{\pi}{t}\right)^{3 / 2} e^{-i \vec{R} \cdot \vec{q}} e^{\frac{1}{4 t}[i \vec{R}+\kappa \hat{z}]^{2}} d t .
$$

Rescaling the integration variable and taking the requisite second derivative with respect to $\kappa$ yields

$$
\beta^{-3 / 2} \int_{1}^{\infty} t^{-1 / 2} e^{-i \vec{R} \cdot \vec{q}} e^{-\pi R^{2} t / \beta v_{W S}^{2 / 3}}\left[-\frac{\pi R_{z}^{2} t^{2}}{\beta v_{W S}^{2 / 3}}+\frac{t}{2}\right] d t .
$$

There is a singular contribution to the total sum from the term in which $\vec{R}=0$. However, that term is independent of $\vec{q}$, and is, therefore, cancelled when the total expression with $\vec{q}=0$ is subtracted. The nonvanishing contribution is the sum over non-zero $\vec{R}$ 's of the expression in (B17). To this is added (B13), and the result is $f(\vec{q})$ defined through (B4).

Note that in all the above, the parameter $\beta$ has not been fixed. It is, in fact, left undetermined, and may be set to speed convergence of the sums. Alternatively, it may be left as an internal check on the procedure, since the final result must be independent of $\beta$.

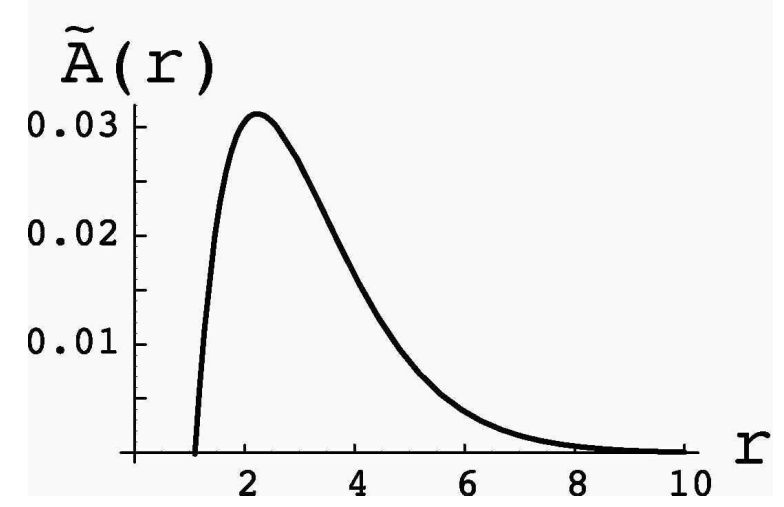

FIG. 7: The quantity $\tilde{A}(r)=2 A(r) /\left(Z^{2} e^{2} / k_{B} T l_{v}\right)$, where $A(r)$ is the coefficient of $k^{2}$ in the quadratic energy of distortion of the modified fcc lattice, quadratic in the displacement field, as a function of the aspect ratio $r=l_{h} / l_{v}$. In this plot, the $z$-component of the wave vector $\vec{q}$ of the distortion has been set equal to zero.

To see what the sum developed above yields, we split the wave vecto $\vec{q}$ as follows: $\vec{q}=\hat{z} Q+\vec{k}$, where the projection, $\vec{k}$, lies in the $x-y$ plane. Numerical results are consistent with an energy quadratic in displacements that is proportional to $k^{2}$ when $Q=0$ and $\vec{k}$ is small. Figure 7 shows the coefficient of $k^{2}$ in that line as a function of the aspect ratio $r=l_{h} / l_{v}$. Note that the coefficient goes through zero at a value of $r$ that is close to 1 . This corresponds to the "spinodal instability" lying below the first-order transition at $r \approx 1.1$. Near the 
spinodal the harmonic spectrum takes the form shown in Eq. [2.9] The coefficient $A(r)$ goes through a maximum as $r$ increases above this value, and then decreases, tending to zero asymptotically as the aspect ratio becomes large. This latter tendency reflects the weakening of the interaction that stabilizes the counterion lattice as the distance between the rods grows in comparison to the distance between neighboring charges on a rod. In fact, it is not hard to demonstrate that $A(r)$ will decay exponentially as $r$, the aspect ratio,gets large.

To deal with terms that are third and fourth order in the displacement field, the procedure is the same as the one discussed above. One is left with lattice sums to perform, and the Ewald method leads to rapidly converging numerical algorithms. The results (2.10) $-(2.13)$ are obtained. We find for the coefficients $B_{1}-B_{3}$ in (2.11), $B_{k}=\left(Z^{2} e^{2} / 2 k_{B} T l_{v}\right) \tilde{B}_{k}$, where

$$
\begin{aligned}
& \tilde{B}_{1}=0.143 \\
& \tilde{B}_{2}=3 \times(-0.163) \\
& \tilde{B}_{3}=0.010
\end{aligned}
$$

\section{APPENDIX C: COUNTERION MELTING IS $3 \mathrm{DXY}$}

As the melting transition is approached from the "counterion liquid" side, we assume that there is an instability leading to a modulated counterion charge density on each rod; then we allow for phase fluctuations. For the counterion density on each rod, we write

$$
\rho(z)=A \cos (\mathcal{Q} z+\phi(z))
$$

Phase fluctuations disorder the charge density wave above and render the mean charge density on a rod statistically uniform in the "liquid" phase. The interaction between counterions on a single rod will be of the form

$$
\frac{1}{2} \iint \rho(z) \rho\left(x^{\prime}\right) V\left(z-z^{\prime}\right) d z d z^{\prime}
$$

Making use of (C1), one obtains terms of the form

$$
\int d z \int d z^{\prime} V\left(z-z^{\prime}\right) \exp \left[i \mathcal{Q}\left(z-z^{\prime}\right)+i\left(\phi(z)-\phi\left(z^{\prime}\right)\right)\right]
$$

We now go to "center of mass" and "relative" coordinates. Let

$$
\begin{aligned}
& Z=\frac{z+z^{\prime}}{2} \\
& \mathcal{Z}=\frac{z-z^{\prime}}{2}
\end{aligned}
$$

The double integral in (C3) is, then, proportional to

$$
\begin{aligned}
& \int d Z \int d \mathcal{Z} V(\mathcal{Z}) \exp \left[i \mathcal{Q Z}+2 i \phi^{\prime}(Z) \mathcal{Z}+\cdots\right] \\
& =\int d Z \int d \mathcal{Z} V(\mathcal{Z}) \exp [i \mathcal{Q Z}] \\
& \quad \times\left(1+2 i \phi^{\prime}(Z) \mathcal{Z}+\frac{1}{2}\left(2 i \phi^{\prime}(Z) \mathcal{Z}\right)^{2}+\cdots\right) \\
& =\int d Z\left[v(\mathcal{Q})-2 i \phi^{\prime}(Z) v^{\prime}(\mathcal{Q})+2\left(\phi^{\prime}(Z)\right)^{2} v^{\prime \prime}(\mathcal{Q})\right]
\end{aligned}
$$

Now, let's assume that

$$
v(q) \propto \int d z \exp [i q z] V(z) d z
$$

has a minimum at $q=\mathcal{Q}$. Then, $v^{\prime}(\mathcal{Q})=0$, and $v^{\prime \prime}(\mathcal{Q})>$ 0 . There is thus a contribution to the total energy going as $\int(d \phi(z) / d z)^{2} d z$.

Now consider the interaction between rods. One expects that there will be terms of the form

$$
\begin{aligned}
& \int d z_{1} \int d z_{2} W\left(z_{1}-z_{2}\right) \\
& \quad \times \exp \left[i \mathcal{Q}\left(z_{1}-z_{2}\right)+i\left(\phi_{1}\left(z_{1}\right)-\phi_{2}\left(z_{2}\right)\right]\right.
\end{aligned}
$$

The $\phi$ 's are subscripted to make it clear that they refer to different rods. If one assumes that the interaction $W$ is sufficiently short-ranged, which seems to be the case even for unscreened Coulomb interactions because of the sinusoidal nature of the assumed state, one reveals the essence by replacing $\phi_{2}\left(z_{2}\right)$ by $\phi_{2}\left(z_{1}\right)$, and the integration in (C8) becomes

$$
\begin{aligned}
& \int d z_{1} \exp \left[i\left(\phi_{1}\left(z_{1}\right)-\phi_{2}\left(z_{1}\right)\right)\right] \\
& \quad \times \int d z_{2} W\left(z_{1}-z_{2}\right) \exp \left[i \mathcal{Q}\left(z_{1}-z_{2}\right)\right] \\
& =w(\mathcal{Q}) \int d z_{1} \exp \left[i\left(\phi_{1}\left(z_{1}\right)-\phi_{2}\left(z_{1}\right)\right)\right] \\
& \quad \rightarrow \int w(\mathcal{Q}) \cos \left[\phi_{1}\left(z_{1}\right)-\phi_{2}\left(z_{1}\right)\right] d z_{1}
\end{aligned}
$$

The result of this heuristic derivation is that there are terms in the energy going as $(d \phi(z) / d z)^{2}$ and that there are also terms going as $\cos \left(\phi_{1}(z)-\phi_{2}(z)\right)$, where the subscripts refer to near-neighbor rods. The universality class for the transition in this model is that of a $3 D X Y$ model. That the model is spatially anisotropic does not influence the universality class. 